\title{
Holographic complexity in general quadratic curvature theory of gravity
}

\author{
Ahmad Ghodsi $^{\mathrm{a}}$, Saeed Qolibikloo ${ }^{\mathrm{b}}$, Saman Karimic \\ Department of Physics, Faculty of Science, Ferdowsi University of Mashhad, Mashhad, Iran
}

Received: 5 July 2020 / Accepted: 26 September 2020 / Published online: 6 October 2020

(C) The Author(s) 2020

\begin{abstract}
In the context of CA conjecture for holographic complexity, we study the action growth rate at late time approximation for general quadratic curvature theory of gravity. We show how the Lloyd's bound saturates for charged and neutral black hole solutions. We observe that a second singular point may modify the action growth rate to a value other than the Lloyd's bound. Moreover, we find the universal terms that appear in the divergent part of complexity from computing the bulk and joint terms on a regulated WDW patch.
\end{abstract}

\section{Introduction}

In recent years, by combining the basic ideas of quantum information theory (QIT) and AdS/CFT duality, a significant development in the area of black hole physics has occurred and we have witnessed the appearance of new paradigms in our quest to understand the quantum theory of gravity.

Holographic entanglement entropy (HEE) is a successful example and has been captured the imagination of many people in this area of research [1,2]. Meanwhile, it was claimed in [3] that the HEE is not enough to distinguish the degrees of freedom inside a black hole, because even if the space-time reaches the thermal equilibrium, the volume of the black hole continues to grow. Instead, complexity is an interesting proposal that characterizes these degrees of freedom.

In QIT, complexity is defined as the minimum number of quantum gates, essential to produce a particular state $|\psi\rangle$ from a reference state $\left|\psi_{0}\right\rangle$. In other words, complexity is a measure of how hard it is to construct a final state from an initial state [4-6].

In the context of AdS/CFT there are two main proposals on how to compute the complexity of a boundary state. The first

\footnotetext{
a e-mail: a-ghodsi@ ferdowsi.um.ac.ir (corresponding author)

be-mail: s.qolibikloo@mail.um.ac.ir

c e-mail: karimi.saman@mail.um.ac.ir
}

one is the "Complexity = Volume" or (CV) conjecture [79] and the second one is the "Complexity=Action" or (CA) conjecture $[10,11]$. The $\mathrm{CV}$ duality states that the complexity of a holographic boundary state on a time slice $\Sigma$ is given by

$C_{V}(\Sigma)=\operatorname{Max}_{\Sigma=\partial B}\left[\frac{V(B)}{G_{N} \ell}\right]$,

where $\ell$ is a certain length scale of the geometry (for example the curvature scale or horizon radius) and $B$ is the corresponding bulk surface. The $\mathrm{CA}$ conjecture claims that the complexity is given by the gravitational action evaluated on the Wheeler-DeWitt (WDW) patch. This patch is defined as the domain of dependence of the Cauchy surface in the bulk which asymptotically approaches the time slice $\Sigma$ on the boundary

$C_{A}(\Sigma)=\frac{I_{\mathrm{WDW}}}{\pi \hbar}$.

In CA conjecture we are dealing with evaluating the gravitational action on various space-time regions including space/time-like boundaries, null boundary surfaces, and various types of joints at the intersection of boundaries [12].

Other than complexity, there are related interesting parameters, which play major roles in the computation of $\mathrm{CV}$ and CA conjectures. For example complexity of formation $\Delta C$, the structure of UV divergences of the complexity, the time dependence or the rate of complexity $d C / d t$, and the socalled Lloyd's bound. In what follows, we briefly review these parameters one by one.

Let us begin by introducing the complexity of formation. The thermofield double (TFD) state can be considered as the dual description of the full geometry of an eternal AdS black hole [13]

$|\mathrm{TFD}\rangle=Z^{-1 / 2} \sum_{i} e^{-E_{i} /(2 T)}\left|E_{i}\right\rangle_{L}\left|E_{i}\right\rangle_{R}$,

where the corresponding asymptotic boundaries (denoted by $\mathrm{L}$ and $\mathrm{R}$ ) are two copies of the same CFT. One can con- 
struct thermal density matrix of the CFT at temperature $T$, by integrating out either the left or right degrees of freedom in the above state. Moreover, the entanglement between these two sets of degrees of freedom leads to the appearance of Einstein-Rosen bridge in the bulk [13,14]. The complexity of formation is the difference between complexity in the process of forming the entangled TFD state and preparing two individual copies of the vacuum state of the left and right boundary CFTs

$\Delta C=\frac{1}{\pi \hbar}[I(\mathrm{BH})-2 I(\mathrm{AdS})]$.

In [15] it was found that for boundary dimensions greater than two, the complexity of formation grows linearly with the thermal entropy at high temperatures $\Delta C \sim S$.

The growth rate of action within the WDW patch at late time approximation or $d C / d t$ is another important factor. One of the interesting features of this parameter is that it tends to a specific universal value. In QIT this limit has been found in the study of an arbitrary quantum system [16] (Lloyd bound). In the context of CA conjecture it was first studied in $[10,11]$, where they show that the bound is violated for large enough Schwarzschild AdS black holes, and is saturated for small ones. Their results suggest that the growth rate would be bounded by the total energy of the system

$$
\frac{d C}{d t} \leq \frac{2 E}{\pi \hbar} .
$$

Further exploration of this bound, in the case of rotating and charged black holes were carried out in $[17,18]$, where a modified formula for the action growth rate was presented. For example in the case of charged black holes

$$
\frac{d I}{d t}=\left(M-\mu_{+} Q\right)-\left(M-\mu_{-} Q\right),
$$

where $M$ and $Q$ are mass and charge of the black hole and $\mu$ is the chemical potential. The \pm signs represent the outer and inner horizons of the black hole.

In the context of gauge/gravity duality, it is usual to consider a given classical theory of gravity with an asymptotically AdS solution as a toy model. Such a model may have not a unique dual CFT but it might probably be a truncation of many various theories with additional fields that have an explicit dual CFT. Therefore by studying the holographic dictionary we explore a family of CFTs in the same universality class. The holographic dual of Einstein gravity is restricted to a specific set of CFTs. To study a broader spectrum of CFTs, one can consider higher curvature theories of gravity in the bulk. This enables us to explore other universality classes as well because as it is observed in these theories there are various types of degrees of freedom in addition to the Einstein modes.
In this regard, the higher order curvature theories of gravity usually have two important roles. Either they show the existence of a certain property that holds for all holographic dual CFTs, for instance, the holographic c-theorems or holographic entanglement entropy, or in opposite direction, they provide counterexamples, for example in the known KovtunSon-Starinets bound for the shear viscosity over entropy density ratio, where the bound is violated in the presence of certain higher curvature terms. These properties motivate us to study the holographic complexity in both directions in this paper. In Sect. 2 we examine possible situations where the Lloyd's bound is preserved or violated and in Sect. 3 we find the universal terms that appear in the divergent part of complexity.

In fact, there has been extensive discussion on the preservation or violation of the Lloyd's bound in various gravitational setups [19-27]. For example in [26], using Noether charge formalism of Iyer and Wald, ${ }^{1}$ the authors have found a modified version of the Lloyd's bound in multiple Killing horizons black hole for a higher curvature theory of gravity. Also, in [30] it was shown that strong energy condition is a sufficient condition to ensure the bound inequality (1.5) and it was argued that the equality (1.6) satisfies the bound (1.5).

Generally, there are two main methods for computation of the action growth rate in CA conjecture, the BRSSZ method introduced in [11], and the LMPS method [12]. In the latter paper, the authors show that the two methods lead to the same result for the action growth rate in Einstein gravity. Also in [31] it was pointed out that even in general higher curvature theories of gravity these two methods provide the same result.

Since in the first approach one does not need to know the corresponding action of the null boundaries in general higher curvature theories of gravity, we use this method to compute the action growth rate. In the BRSSZ method for the computation of $d C / d t$, in addition to the bulk action, it is only necessary to know the action on the time/space-like boundaries, which is essentially the Gibbons-Hawking-York (GHY) action and its generalizations (see Sect. 2 for more details).

For a general higher curvature gravitational theory it is hard to find an appropriate surface term to make the variational principle well-posed [32], but the non-null surface terms have been developed for some gravitational theories, such as $F(R)$, Gauss-Bonnet gravity and Lanczos-Lovelock theory [33-38] and other higher curvature theories [39]. Specifically for $f$ (Riemann) theories of gravity by using the auxiliary field formalism these surface terms have been investigated in [40]. In this context, complexity has been studied for higher curvature theories of gravity of example see [41-45]. Inspired by this, we are going to consider the

\footnotetext{
$\overline{{ }^{1} \text { See also }[28,29]}$.
} 
general quadratic curvature (GQC) theory of gravity in this paper and compute the action growth rate in this theory.

Another related subject is the structure of UV divergences of the complexity. In both complexity conjectures, we evaluate quantities which diverge by extending near the asymptotic boundaries. These divergences are related to the existence of short-scale correlations in the dual boundary CFTs. As it was shown in [46] the coefficients in these divergent terms can be written in terms of intrinsic and extrinsic boundary curvatures. The general structure of divergent terms in complexity in $d$ dimensional space-time can be written as

$$
\begin{gathered}
C_{A} \sim \frac{a_{1}}{\delta^{d-2}}+\frac{a_{2}}{\delta^{d-4}}+\cdots+\log \left(\frac{\ell}{\delta}\right) \\
\times\left[\frac{b_{1}}{\delta^{d-2}}+\frac{b_{2}}{\delta^{d-4}}+\cdots\right],
\end{gathered}
$$

where $\delta$ is the UV cut-off. Here the coefficients $a_{i}$ depend on the regularization scheme but the coefficients $b_{i}$ are universal (regulator independent). In Sect. 3 we will study the structure of these divergences of the complexity and compute the universal terms of the complexity in GQC gravity.

\section{The action growth rate of GQC gravity}

Following $[11,17]$ we are going to compute the action growth rate on a WDW patch associated with a two-sided black hole in GQC theory of gravity, see Fig. (1). It is believed that this would be dual to the rate of growth of the complexity of the boundary state. This black hole is a charged/neutral Schwarzschild AdS solution of the equations of motion. For charged solution (left diagram) we suppose outer and inner horizons at $r=r_{ \pm}$. For neutral case (right diagram) we consider inside the black hole is limited from singularity to the future horizon.

According to the arguments of [11], the WDW patch is bounded by $t_{L}$ and $t_{R}$ on the left and right for a (un)charged black hole. When time passes on the left boundary from $t_{L}$ to $\bar{t}_{L}$, the WDW patch starts growing in the green region and shrinks in the red region. At late times, the whole contribution of action growth comes from the green region that now lives between inner and outer horizons at $r=r_{ \pm}$of the charged black hole or between singularity at $r=r_{s}$ and future horizon of the neutral black hole. Therefore the total contribution of action comes from the bulk action in this region as well as the boundary actions on the space-like surfaces at $r_{ \pm}$or the singularity and future horizon.

\subsection{General quadratic curvature action}

Let us first introduce the bulk and boundary actions of GQC. The bulk action consists of the Einstein-Hilbert action together with a cosmological term. Moreover, we add all quadratic curvature terms as the sum of Ricci and Ricci scalar curvature squared terms and the well-known Gauss-Bonnet (GB) terms. To study charged black holes we also consider a $U(1)$ gauge field through a Maxwell term in our Lagrangian

$$
\begin{aligned}
I_{\text {bulk }}= & -\frac{1}{2 \kappa^{2}} \int_{\mathcal{M}} d^{d} x \sqrt{-g} \\
& \times\left(R-2 \Lambda_{0}+a_{1} R^{2}+a_{2} R_{\mu \nu}^{2}+a_{3}\right. \\
& \left.\times\left(R^{2}-4 R_{\mu \nu}^{2}+R_{\mu \nu \rho \sigma}^{2}\right)-F_{\mu \nu} F^{\mu \nu}\right),
\end{aligned}
$$

where $\kappa^{2}=8 \pi G$. To write the action for boundary surfaces, which make the variational principle of the gravitational field well-defined, instead of using the auxiliary field formalism (see e.g. [40]), we consider three types of Gibbons-Hawking (GH) terms corresponding to the Einstein-Hilbert, GB and Ricci square terms. The GH term associated to the EinsteinHilbert action is given by

$I_{G H}^{E H}=-\frac{1}{\kappa^{2}} \int_{\partial \mathcal{M}} d^{d-1} x \sqrt{-h} K$,

where $h$ is the determinant of the induced metric on the boundary surfaces $\partial \mathcal{M}$ and $K$ is the trace of the extrinsic curvature. The extrinsic curvature is defined by

$K_{\mu \nu}=\frac{1}{2}\left(\nabla_{\mu} n_{\nu}+\nabla_{\nu} n_{\mu}\right)$,

and $n_{\mu}$ is a space-like unit vector, normal to the boundary. Moreover, there is a generalized GH action corresponding to the GB gravity [36]

$$
\begin{aligned}
I_{G H}^{G B}= & \frac{2}{\kappa^{2}} a_{3} \int_{\partial \mathcal{M}} d^{d-1} x \sqrt{-h} \\
& \times\left(2 \mathcal{G}_{a b} K^{a b}+\frac{1}{3}\left(K^{3}-3 K K_{a b} K^{a b}+2 K_{a b} K^{b c} K_{c}{ }^{a}\right)\right),
\end{aligned}
$$

where $\mathcal{G}_{a b}$ is the Einstein tensor constructed out of the induced metric. For Ricci squared terms of the action we use the following expression for GH term

$I_{G H}^{R i c^{2}}=-\frac{1}{\kappa^{2}} \int_{\partial \mathcal{M}} d^{d-1} x \sqrt{-h} \frac{\partial L^{R i c^{2}}}{\partial R_{\alpha \mu \beta \nu}} n_{\mu} n_{\nu} K_{\alpha \beta}$.

The total GH term therefore is the sum of these three parts

$I_{G H}=I_{G H}^{E H}+I_{G H}^{G B}+I_{G H}^{R i c^{2}}$.

\subsection{Charged solution in d dimension}

To find a charged solution with asymptotic AdS symmetries we use the following metric in $d$ dimensional space-time

$$
d s^{2}=-f_{1}(r) d t^{2}+f_{2}^{-1}(r) d r^{2}+r^{2} d \Omega_{d-2}^{2} .
$$


Fig. 1 Penrose diagram of charged (left) and neutral (right) black holes in GQC gravity. In the charged case we have depicted the outer horizon $r_{+}$ and the inner horizon $r_{-}$, together with two singularities, one at the center of space-time $r=0$, and one behind the inner horizon, at $r=r_{s}$, denoted by the wiggly arcs in this picture. In the neutral case, however, the inner horizon disappears and space-like singularities appear, therefore the WDW patch should be ended on the singularity

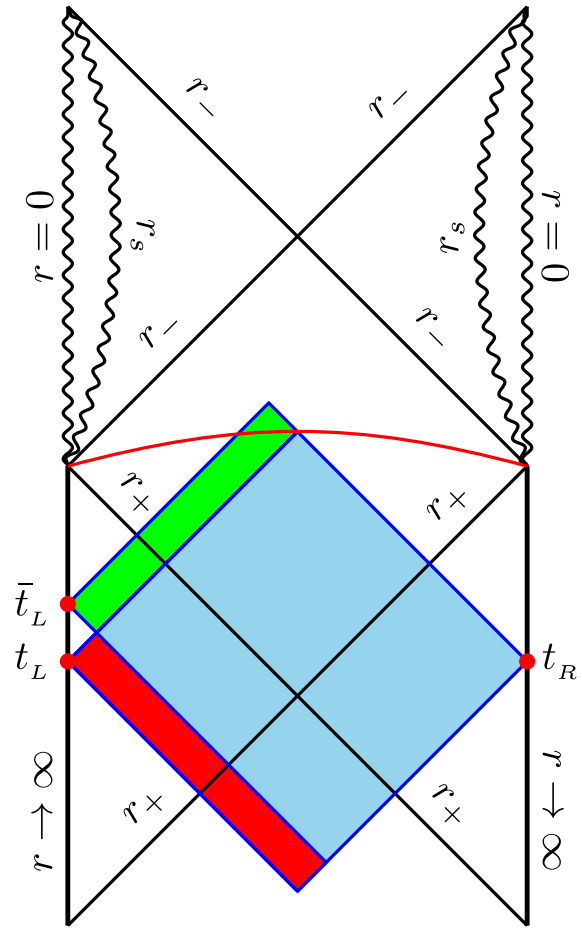

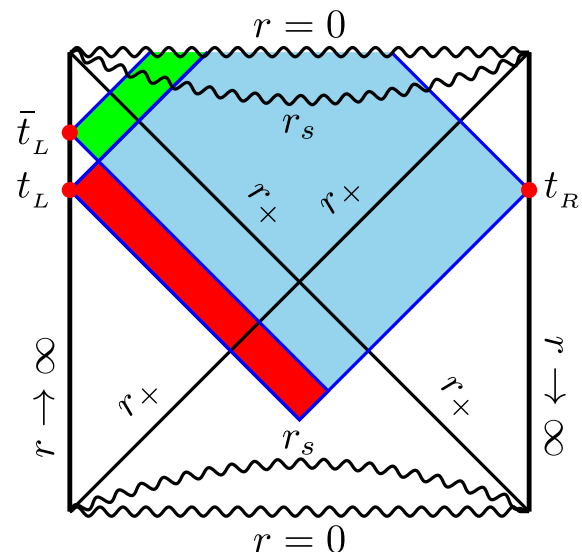

The field strength satisfies the Maxwell equation in $d$ dimension, i.e. $\nabla_{\mu}\left(\sqrt{-g} F^{\mu \nu}\right)=0$

$$
F_{t r}=-\sqrt{\frac{1}{2}(d-2)(d-3)} \frac{q}{r^{d-2}} \sqrt{\frac{f_{1}(r)}{f_{2}(r)}},
$$

where $q$ is the constant of integration, and is related to the electric charge $Q$ by

$q^{2}=\frac{\kappa^{2}}{(d-2) \Omega_{d-2}} Q^{2}$.

By variation of the Lagrangian (2.1) and inserting (2.7) and (2.8) for metric and field strength, we will find a couple of third and fourth order differential equations for $f_{1}(r)$ and $f_{2}(r)$. Finding an exact solution is a hard task to do, instead we find a solution which is linear in terms of the couplings of theory i.e. $a_{1}, a_{2}$ and $a_{3}$. The solution is given by

$$
\begin{aligned}
f_{1}(r)= & 1-\frac{2 \Lambda_{0}}{(d-1)(d-2)}\left(1-\frac{C_{0} \Lambda_{0}}{(d-2)^{2}(d-1)}\right) r^{2} \\
& +q^{2}\left(1-\frac{4 \Lambda_{0} C_{q}}{(d-2)(d-1)}\right) \frac{1}{r^{2 d-6}} \\
& -\frac{m}{r^{d-3}}+\frac{d-3}{d-2} \\
& \times\left(C_{1} \frac{1}{r^{2 d-4}}+2 q^{2} m C_{2} \frac{1}{r^{3 d-7}}+q^{4} \frac{C_{3}}{(3 d-7)} \frac{1}{r^{4 d-10}}\right),
\end{aligned}
$$

$\frac{f_{2}(r)}{f_{1}(r)}=h(r)$,

$$
\begin{aligned}
h(r)= & 1-4 \frac{d-3}{d-2} \\
& \times\left(a_{1}(d-4)(2 d-3)+a_{2}\left(d^{2}-5 d+5\right)\right) q^{2} r^{4-2 d,}
\end{aligned}
$$

where all the above coefficients are as follow

$$
\begin{aligned}
C_{q}= & a_{3}(d-4)(d-3)+a_{1} d(d-1)-a_{2}(d(d-6)+7), \\
C_{0}= & 2(d-4)\left(a_{3}(d-3)(d-2)+a_{2}(d-1)+a_{1} d(d-1)\right), \\
C_{1}= & \left(4 a_{1}(d-4)-2 a_{2}(d(d-6)+10)\right) q^{2} \\
& +a_{3}(d-4)(d-2) m^{2}, \\
C_{2}= & -a_{1}(d-4)(d-1)+a_{2}-a_{3}(d-4)(d-2), \\
C_{3}= & (d-4)\left(a_{3}(d-2)(3 d-7)+a_{1}\left(11 d^{2}-45 d+44\right)\right) \\
& +a_{2}\left(4 d^{3}-33 d^{2}+83 d-64\right) .
\end{aligned}
$$

To prevent the divergence in the gauge field strength (2.8), the function $h(r)$ in relation (2.10b) should not be equal to zero. This requires considering the following condition between couplings of the theory

$a_{1}(d-4)(2 d-3)+a_{2}\left(d^{2}-5 d+5\right) \leq 0$.

For the neutral solution as we send $q \rightarrow 0$, both functions in (2.10a) and (2.10b) are equal or $h(r)=1$ at this level of perturbation, but it can be shown that, $f_{1}(r)$ and $f_{2}(r)$ are separated when we consider next orders of perturbation into account.

\subsection{Action growth rate in WDW patch}

The growth rate of bulk action in the WDW patch at late-time approximation can be computed by inserting the solution 
(2.10a) and (2.10b) into bulk action (2.1). For more details of computations see relations (A.1a)-(A.1c) in Appendix A

$$
\begin{aligned}
\frac{d I_{b u l k}}{d t}= & \frac{\Omega_{d-2}}{2 \kappa^{2}} \int_{r_{-}}^{r_{+}} d r \frac{2 r^{-2-3 d}}{(d-2)^{3}(d-1)} \\
& \times\left(\alpha_{1} r^{2 d+4}+\alpha_{2} r^{2 d+2}+\alpha_{3} r^{d+5}+\alpha_{4} r^{4 d}+\alpha_{5} r^{8}\right),
\end{aligned}
$$

where $\alpha_{1}, \ldots, \alpha_{5}$ are given in Eq. (B.1) in Appendix B. In calculation of action growth in this section we consider $r_{ \pm}$ as outer/inner horizons and we suppose that there is no singularity in between them.

At late-time approximation, the growth rate of GibbonsHawking surface terms of WDW patch is obtained by computing the value of (2.6). To do this we insert the solution (2.10a) and (2.10b) into the Eqs. (A.2) and (A.3) and we find

$$
\begin{aligned}
\frac{d I_{G H}}{d t}= & -\frac{\Omega_{d-2}}{\kappa^{2}} \frac{1}{6(d-2)^{3}(d-1)(3 d-7)} \\
& \times\left(\beta_{1} r^{-3 d+7}+\beta_{2} r^{d-1}+\beta_{3} r^{d-3}\right. \\
& +\beta_{4} r^{d-5}+\beta_{5}+\beta_{6} r^{-2}+\beta_{7} r^{-d+3} \\
& \left.+\beta_{8} r^{-d+1}+\beta_{9} r^{-2 d+4}\right)\left.\right|_{r_{-}} ^{r_{+}}
\end{aligned}
$$

where $\beta_{1}, \ldots, \beta_{9}$ are given in Eq. (B.2). Finally, we perform the integration of (2.13) and add it to the relation (2.14) which leads to the total action growth rate. The final result can be simplified by using the following steps:

1. Because we considered $r_{+}$as the outer horizon, we can solve $f_{1}\left(r_{+}\right)=0$ to find a relation for $\Lambda_{0}$.

2. Insert the value of $\Lambda_{0}$ from the first step into $f_{1}\left(r_{-}\right)=0$ to find a relation for $m$ in terms of $r_{+}$and $r_{-}$.

3. Insert the relations of $\Lambda_{0}$ and $m$ in the first and second steps into the total action growth rate. The final result is

$$
\begin{aligned}
\frac{d I_{\text {tot }}}{d t}= & -\frac{\Omega_{d-2}}{\kappa^{2}} q^{2} \\
& \times\left((d-2)\left(\frac{1}{r_{-}^{d-3}}-\frac{1}{r_{+}^{d-3}}\right)+\frac{2(d-3)^{2} q^{2}}{3 d-7}\right. \\
& \times\left(a_{1}(2 d-3)(d-4)+a_{2}\left(5-5 d+d^{2}\right)\right) \\
& \left.\times\left(\frac{1}{r_{-}^{3 d-7}}-\frac{1}{r_{+}^{3 d-7}}\right)\right)
\end{aligned}
$$

Using the field strength relation (2.8) and the value of electric charge in (2.9), one can write the above expression as follow $\frac{d I_{t o t}}{d t}=\left(M-\mu_{+} Q\right)-\left(M-\mu_{-} Q\right)$, where we have supposed $M$ as the mass of black hole and

$$
\begin{aligned}
\mu_{ \pm}= & -\frac{Q}{r_{ \pm}^{d-3}}-\frac{\kappa^{2}}{\Omega_{d-2}} \frac{2(d-3)^{2}}{(d-2)^{2}(3 d-7)} \\
& \times\left(a_{1}(2 d-3)(d-4)+a_{2}\left(5-5 d+d^{2}\right)\right) \\
& \times \frac{Q^{3}}{r_{ \pm}^{3 d-7}},
\end{aligned}
$$

are the values of chemical potential on $r_{ \pm}$horizons.

The result of Eq. (2.16) shows that the proposal introduced in [17] is correct for general quadratic curvature theory. Using the Noether charge formalism of Iyer and Wald [26] finds this modified version of the Lloyd's bound in multiple Killing horizons black hole for a higher curvature theory of gravity.

\subsection{Neutral black hole in d dimension}

To find the action growth rate in the neutral case we need to know the singular points of the geometry. To do this we first review the solutions of equations of motion for some special cases:

- $a_{3}=0$ : In this case the exact solution of equations of motion is given by the known Schwarzschild AdS black hole, for example see [49]

$$
\begin{aligned}
& f_{1}(r)=f_{2}(r)=1+\frac{r^{2}}{L^{2}}-\frac{m}{r^{d-3}}, \\
& -(d-2)(d-1) \frac{1}{L^{2}}+(d-4)(d-1)^{2} \\
& \quad \times\left(a_{2}+a_{1} d\right) \frac{1}{L^{4}}-2 \Lambda_{0}=0 .
\end{aligned}
$$

This solution has a singularity at $r_{s}=0$.

- $a_{1}=a_{2}=0$ : This case corresponds to the Gauss-Bonnet gravity. The exact solution is given by [17]

$$
\begin{aligned}
f_{1}(r)= & f_{2}(r)=1+\frac{r^{2}}{\lambda} \\
& \times\left(1-\sqrt{\left.1+2 \lambda\left(\frac{2 \Lambda_{0}}{(d-1)(d-2)}+\frac{m}{r^{d-1}}\right)\right)},\right. \\
\lambda= & 2 a_{3}(d-4)(d-3) .
\end{aligned}
$$

This solution has two singularities. The first one, is where the metric is divergent and the other one, is the point where the metric or scalar curvature terms become imaginary

$r_{s}=0, \quad r_{s}=\left(-\frac{2 \lambda(d-2)(d-1)}{(d-1)(d-2)+4 \lambda \Lambda_{0}} m\right)^{\frac{1}{d-1}}$

The above analysis for special cases helps us to find the singularity structure of the solution for general case. To make 
the analysis easier, it would be better to find a solution which reduces to solution (2.10a) when $q \rightarrow 0$ and reduces to (2.19a) when $a_{1}=a_{2}=0$.

In fact, we may assume that there is a general solution, which can be written as $f(r)=1+r^{2} X(r)(1-$ $\left.\sqrt{1+c+\frac{m}{r^{d-1}}}\right)$ and solve equations of motion perturbativly to find $c, m$ and $X(r)$. To first order of perturbation both $f_{1}(r)$ and $f_{2}(r)$ functions are given by

$$
\begin{aligned}
f(r)= & 1+\frac{1-\frac{2(d-4)\left(a_{2}+d a_{1}\right) \Lambda_{0}}{(d-2)^{2}} r^{2}}{\lambda} \\
& \times\left(1-\sqrt{1+2 \lambda\left(\frac{2 \Lambda_{0}}{(d-1)(d-2)}+\frac{m}{r^{d-1}}\right)}\right) .
\end{aligned}
$$

We can see that (2.21) has the same the singularity structure as (2.19a) at least at this order of perturbation. By an expansion around small couplings (2.21) would be equal to

$$
\begin{aligned}
f(r)= & 1+\frac{r^{2}}{L^{2}}-\frac{m}{r^{d-3}}+\frac{\lambda}{2} \frac{m^{2}}{r^{2 d-4}}, \\
\frac{1}{L^{2}}= & -\frac{2 \Lambda_{0}}{(d-1)(d-2)} \\
& \times\left(1-\frac{(d-2) \lambda+2(d-1)(d-4)\left(a_{2}+d a_{1}\right)}{(d-2)^{2}(d-1)} \Lambda_{0} \times\right),
\end{aligned}
$$

where in this expansion $m=m_{0}+\delta m$ ( $m_{0}$ is a constant of integration) and

$$
\begin{aligned}
\delta m= & \frac{-2}{(d-2)^{2}(d-1)} \\
& \times\left((d-2) \lambda+(d-1)(d-4)\left(a_{2}+d a_{1}\right)\right) \Lambda_{0} m_{0} .
\end{aligned}
$$

\subsection{Action growth rate in WDW patch}

To find the growth rate of actions for neutral Schwarzschild AdS black hole we carry out the following steps:

1. First, we add Eqs. (2.13) and (2.14) after taking the limit of $q \rightarrow 0$, with a distinction that the upper limit is $r=r_{h}$, the location of the future horizon and the lower limit is $r=r_{s}$, the location of the singularity

$$
\begin{aligned}
\frac{d I_{t o t}^{q=0}}{d t}= & \frac{\Omega_{d-2}}{6 \kappa^{2}} \\
& \times\left(3(d-1) m-(d-2) m^{2} r^{1-d} \lambda+2 m\right. \\
& \times\left(\frac{(d-2) \lambda}{r^{2}}+\frac{6(d-1)\left(a_{2}+a_{1} d\right) \Lambda_{0}}{d-2}+\frac{\lambda \Lambda_{0}}{d-4}\right)
\end{aligned}
$$

$$
\begin{aligned}
& +\frac{2 r^{d-5}}{(d-1)^{2}(d-2)^{2}}\left(2(d-2)^{2}(d-1) r^{2}\right. \\
& \times\left(3 r^{2}+\lambda-6(d-1)\left(a_{2}+a_{1} d\right)\right) \Lambda_{0} \\
& \times-(d-2)^{3}(d-1)^{2}\left(3 r^{2}+2 \lambda\right)+2 r^{4} \\
& \left.\left.\times\left(6(d-1) d\left(a_{2}+a_{1} d\right)-(d-2) \lambda\right) \Lambda_{0}^{2}\right)\right)\left.\right|_{r_{s}} ^{r_{h}}
\end{aligned}
$$

2. The horizon is given by solving the equation

$$
1+\frac{r_{h}^{2}}{L^{2}}-\frac{m}{r_{h}^{d-3}}+\frac{\lambda}{2} \frac{m^{2}}{r_{h}^{2 d-4}}=0 .
$$

We use this equation as a constraint between $r_{h}$ and other parameters. Suppose that the location of horizon from this equation can be found perturbativly by putting $r_{h}=$ $r_{0}+\delta r$ into (2.25). In this way, we can find the following relations

$$
\begin{aligned}
\delta r= & \frac{1}{-(d-3) m_{0} r_{0}^{2-d}+\frac{4 r_{0} \Lambda_{0}}{(d-1)(d-2)}} \\
& \times\left(\frac{1}{2} \lambda m_{0}^{2} r_{0}^{4-2 d}-\delta m r_{0}^{3-d}\right. \\
& \left.+\frac{2\left(\lambda(d-2)+2(d-1)(d-4)\left(a_{2}+a_{1} d\right)\right) r_{0}^{2} \Lambda_{0}^{2}}{(d-2)^{3}(d-1)^{2}}\right), \\
1- & m_{0} r_{0}^{3-d}-\frac{2 r_{0}^{2} \Lambda_{0}}{(d-1)(d-2)}=0 .
\end{aligned}
$$

3. Using the above equations we can simplify the growth rate (2.24) at $r=r_{h}$. For simplicity from now on we drop the constant (independent of $r_{h}$ ) terms in (2.24) (note that they will cancel when we compute the total growth), then we have

$$
\begin{aligned}
\left.\frac{d I_{t o t}^{q=0}}{d t}\right|^{r_{h}}= & -\frac{m \Omega_{d-2}}{\kappa^{2}} \\
& \times\left((d-2)+\frac{2(d-3) \Lambda_{0}}{(d-1)(d-2)}\right. \\
& \left.\times\left(a_{3}(d-5)(d-2)+(d-1)\left(a_{2}+a_{1} d\right)\right)\right) .
\end{aligned}
$$

4. In general the action growth rate at $r=r_{s}$ diverges when $r_{s} \rightarrow 0$. Nevertheless, there are special cases that its value is finite:

- For $a_{3}=0$, as we already mentioned, the singularity is located at $r_{s}=0$. At this value the Eq. (2.24) will be equal to

$\left.\frac{d I_{t o t}^{q=0}}{d t}\right|_{r_{s}=0}=\frac{2 m \Omega_{d-2}}{\kappa^{2}} \frac{d-1}{d-2}\left(a_{2}+a_{1} d\right) \Lambda_{0},(2.28)$ therefore the total action growth rate would be equal to 


$$
\begin{aligned}
\frac{d I_{\text {tot }}^{q=0}}{d t} & =-\frac{m \Omega_{d-2}}{\kappa^{2}}\left((d-2)+4\left(a_{2}+a_{1} d\right) \Lambda_{0}\right) \\
& =2 M .
\end{aligned}
$$

The last equality is written in terms of the mass of black hole for the Ricci square theory of gravity. For example see [49] for more details of computation of this mass. ${ }^{2}$ Note that (2.29) is a general result and for example as a special case it gives the result of $d$ dimensional critical gravity.

- For $d=4$ the Gauss-Bonnet part of the theory is a topological term. The growth rate in this case is equal to

$$
\begin{aligned}
\frac{d I_{\text {tot }}^{q=0}}{d t}= & -\frac{4 \pi^{2} m}{\kappa^{2}}\left(\left(2+4 a_{1}+a_{2}-\frac{2}{3} a_{3}\right)\right. \\
& \left.-\left(-12 a_{1}-3 a_{2}-\frac{2}{3} a_{3}\right)\right) \\
= & -\frac{8 \pi^{2} m}{\kappa^{2}}\left(1+2\left(a_{2}+4 a_{1}\right) \Lambda_{0}\right)=2 M .
\end{aligned}
$$

5. For a more general case when $a_{3}<0$, there is a second singularity at $r_{s} \neq 0$. This can be seen from Eq. (2.20) for $m>0$ and $\Lambda_{0}<0$. Although this new point of singularity makes the Eq. (2.24) finite, but the final result leads to a wrong answer for late time complexity

$$
\begin{aligned}
\frac{d I_{t o t}^{q=0}}{d t}= & -\frac{(d-2) \Omega_{d-2}}{\kappa^{2}} \\
& \times\left(\frac{13}{12} m+\frac{7}{3 \times 4^{d-1}}\right. \\
& \left.\times\left(-2(d-3)(d-4) a_{3} m\right)^{\frac{d-3}{d-1}}\right) .
\end{aligned}
$$

A similar behavior also has been reported for the $d=5$ dimensional Gauss-Bonnet gravity in [17].

\subsection{More on the second singularity and complexity}

In previous sections we studied the GQC black hole's solution perturbativly. We assumed two outer and inner horizons and singularity was located behind the inner one. As we investigated (perturbativly) in neutral black holes, it is possible to have a second singularity which leads to a wrong answer for late time complexity. In this section we will try to study $d$-dimensional charged black hole solution in GaussBonnet gravity which can be found analytically and contains a second singularity as well, (see [17] for $d=5$ ). The metric (2.7) after solving the equations of motion is given by

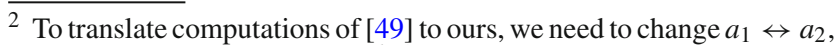
$\kappa^{2} \rightarrow-\kappa^{2}$ and replace $l=1, r_{0}^{d-1}=m, V_{d-2}=\Omega_{d-2}, \bar{\sigma}=1+2(d-$ 1) $\Lambda\left(a_{2}+a_{1} d\right)$ and $\Lambda_{0}=\frac{1}{2}(d-1) \Lambda\left(d-2+(d-4)(d-1) \Lambda\left(a_{2}+d a_{1}\right)\right)$ in mass equation (5.13) i.e. $M=\frac{(d-2) V_{d-2}}{8 \pi G_{d} l}\left(\frac{r_{0}}{l}\right)^{d-1} \bar{\sigma}$.

$$
\begin{aligned}
& f_{1}(r)=f_{2}(r)=f(r) \\
& f(r)=1+\frac{r^{2}}{\lambda}
\end{aligned}
$$

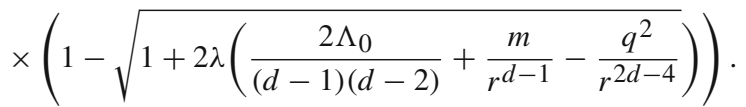

Then the location of horizons and the second singularity (interval) are given by the following algebraic equations

$f\left(r_{h}\right)=0,1+2 \lambda\left(\frac{2 \Lambda_{0}}{(d-1)(d-2)}+\frac{m}{r_{s}^{d-1}}-\frac{q^{2}}{r_{s}^{2 d-4}}\right)=0$.

In general, finding the analytic solutions for above equations is a hard task to do, but a simple numerical investigation shows the possible situations. In Fig. (2) we have sketched the various possible solutions for $f(r)$.

The asymptotic AdS nature of solution restricts us to consider $\Lambda_{0}<0$. Moreover, $m$ is proportional to the mass of black hole so $m>0$. The Gauss-Bonnet coupling could be either negative or positive. In Fig. 2 we have fixed $\Lambda_{0}=$ $-0.01, m=1$ and $a_{3}=\mp 0.02$ in the left/right diagrams, in $d=5$. For various values of the charge $q$, we have the following behaviors:

The left diagram:

1. There is a naked singularity at $r_{s}=0$, green curve.

2. There are two horizons and a singularity at $r_{s}=0$, orange curve.

3. A second singularity at $r_{s} \neq 0$ exists between two horizons, red and blue curves.

4. The second singularity at $r_{s} \neq 0$ exists behind the horizons, black curve (neutral solution).

The right diagram:

1. A singularity at $r_{s} \neq 0$ exists behind the inner horizon, green to blue curves.

2. A solution with a horizon and no singularity, black curve (neutral solution).

By performing the same steps as we did in the previous sections we find the following relation for total action growth:

When the second singularity is behind the inner horizon the situation is similar to the perturbed solution which we found already

$$
\begin{aligned}
\frac{d I_{t o t}}{d t} & =\frac{\Omega_{d-2}}{\kappa^{2}} q^{2}(d-2)\left(\frac{1}{r_{+}^{d-3}}-\frac{1}{r_{-}^{d-3}}\right) \\
& =\left(M-\mu_{+} Q\right)-\left(M-\mu_{-} Q\right) .
\end{aligned}
$$

On the other hand when the second singularity is between the two horizons

$\frac{d I_{t o t}}{d t}=\frac{\Omega_{d-2}}{\kappa^{2}}$ 

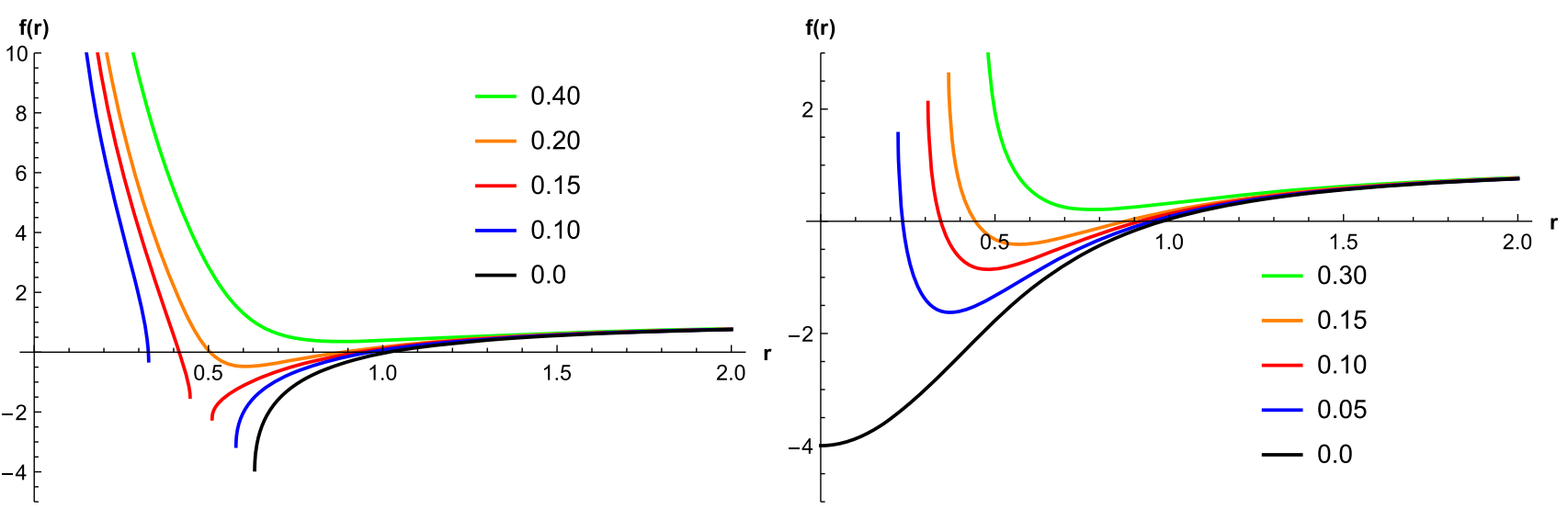

Fig. 2 Horizon and singularity structure of charged Gauss-Bonnet black holes

$$
\times\left(q^{2}\left(\frac{1}{r_{h}^{d-3}}-\frac{1}{r_{s}^{d-3}}\right)+\frac{2(d-2) r_{s}^{d-5}}{3 \lambda}\left(r_{s}+\lambda\right)^{2}\right) .
$$

As we see, although the first term can be written as $(M-$ $\left.\mu_{h} Q\right)-\left(M-\mu_{s} Q\right)$, but there is an extra term which leads us to another value for the Lloyd's bound.

\section{WDW action for global AdS}

In this section, we are going to compute the universal terms that appear in the divergent part of $C_{A}$ complexity in the GQC theory of gravity. All steps that we follow here, have been presented already in reference [47]. We will show how with some simple modifications we can find the universal coefficients of $C_{A}$.

Paper [47] begins with Lovelock theory with the following bulk and space/time-like boundary actions

$I=\sum_{n} \lambda_{n}\left(\int_{M} d^{d} x \sqrt{-g} \mathcal{X}_{2 n}+\int_{\cup_{k} B_{k}} d^{d-1} x d \Sigma \mathcal{Q}_{n}\right)$

where $\mathcal{X}_{2 n}$ is the Euler density and $\mathcal{Q}_{n}$ is the generalized GH boundary term. The boundary terms make the variational principle well defined. For example for $n=1,2$, the relations for $\mathcal{Q}_{1}$ and $\mathcal{Q}_{2}$ are given in Eqs. (2.2) and (2.4).

After that, [47] computes the contribution from space/timelike joints by employing the Hayward smoothing method. These joints are co-dimension two surfaces, which are made from the intersection of boundary surfaces. In this way [47] prove that the joint terms can be computed from the Lovelock boundary term in (3.1) and are given by

$I_{\text {joint }}=\sum_{n} \lambda_{n} \int_{C} d \sigma 2 n \eta \hat{\mathcal{X}}_{2(n-1)}$,

where $\eta= \pm \cosh ^{-1}\left|n_{1} . n_{2}\right|$, and $n_{1,2}$ are normal one-forms to each boundary that their intersection makes the joint $C$.
In above equation $\hat{\mathcal{X}}_{2(n-1)}$ is the Euler density constructed from the induced metric on the joint.

To find the universal terms, one needs to compute the gravitational action on a regularized WDW patch. This patch contains a cut-off distance $\delta$ from the boundary (see Fig. (3))

By choosing this patch, [47] argues that despite of surface terms for null boundaries, the universal terms are originating either from the joint terms (3.2) or the bulk action. The final form of joint term is given by

$I_{\text {joint }}=-\frac{1}{\kappa^{2}} \int_{C} d \sigma a\left[1+\sum_{n=2}^{[(d-1) / 2]} n \lambda_{n} \hat{\mathcal{X}}_{2(n-1)}\right]$,

where $a= \pm \log \left|k_{1} \cdot k_{2} / 2\right|$ for joints between two null boundaries and $a= \pm \log \left|n \cdot k_{1}\right|$ for joints between a timelike and a null boundary.

Now consider the GQC action (2.1) without Yang-Mills term. Although the bulk action cannot be written as a sum of Euler terms but as it was shown in [48] or from computing Eq. (2.5) for a Global AdS solution, the total GH boundary term (2.6) can be written as

$I_{G H}=\lambda_{e f f} I_{G H}^{E H}+I_{G H}^{G B}, \quad \lambda_{e f f}=1+4 \Lambda_{0} \frac{d a_{1}+a_{2}}{d-2}$.

Therefore the joint term (3.3) for GQC theory simplifies to

$I_{\text {joint }}^{G Q C}=-\frac{1}{\kappa^{2}} \int_{C} d \sigma a\left[\lambda_{\text {eff }}+2 a_{3} \hat{\mathcal{R}}\right]$,

where $\hat{\mathcal{R}}$ is the scalar curvature on the joint that is constructed from the induced metric.

Following [46], we use the regulated graph depicted in Fig. (3), i.e. we change the WDW patch by an inward shift on the right and left edges. To compute the structure of divergences, we begin with the following metric which is asymptotically $A d S_{d}$ space-time with radius $\tilde{L}$ in GQC theory

$$
d s^{2}=-f(r) d t^{2}+f^{-1}(r) d r^{2}+r^{2} d \Omega_{d-2}^{2},
$$


Fig. 3 Two ways for regularizing the WDW patch
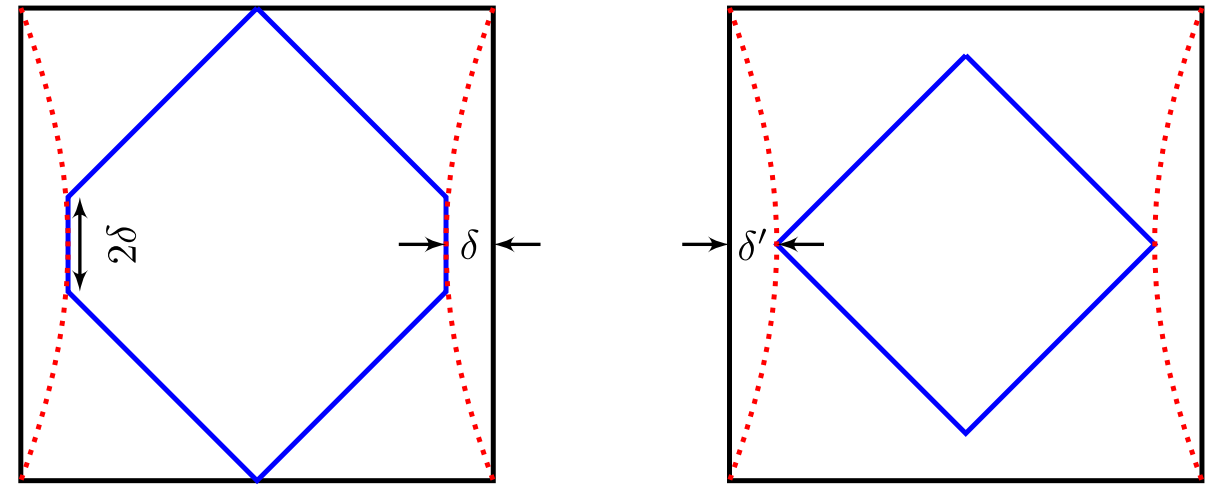

$\left.f(r)\right|_{r \rightarrow \infty} \approx 1+\frac{r^{2}}{\tilde{L}^{2}}$,

$(3.6 \mathrm{a})$

$$
\begin{aligned}
\frac{1}{\tilde{L}^{2}}= & \frac{-2 \Lambda_{0}}{(d-1)(d-2)}\left(1-\frac{2 \Lambda_{0}(d-4)}{(d-1)(d-2)^{2}}\right. \\
& \left.\times\left((d-1)\left(d a_{1}+a_{2}\right)+a_{3}(d-2)(d-3)\right)\right) .
\end{aligned}
$$

By two successive proper coordinate transformation i.e. $z=$ $\frac{\tilde{L}}{r}$ and $t=\tau \tilde{L}$, and then $z=\frac{2 \tilde{L} \cos \theta}{1+\sin \theta}$ we find the following metric

$d s^{2}=\frac{\tilde{L}^{2}}{\cos ^{2} \theta}\left(-d \tau^{2}+d \theta^{2}+\sin ^{2} \theta d \Omega_{d-2}^{2}\right)$.

In this metric, the null boundaries of the WDW patch (the right diagram of Fig. (3)) are given by

$S^{+}: \theta=\frac{\pi}{2}-\tau-\delta^{\prime} \quad$ for $\quad \frac{\pi}{2}-\delta^{\prime} \geq \tau \geq 0$,

$S^{-}: \theta=\frac{\pi}{2}+\tau-\delta^{\prime} \quad$ for $\quad-\frac{\pi}{2}+\delta^{\prime} \leq \tau \leq 0$.

Moreover the unit normal vectors to $S^{+}$and $S^{-}$null surfaces are

$k_{1}=\alpha_{1} \tilde{L}(d \theta+d \tau), \quad k_{2}=\alpha_{2} \tilde{L}(d \theta-d \tau)$,

where $\alpha_{1,2}$ are normalization constants. By another change of coordinate, $\theta^{\prime}=\frac{\pi}{2}-\theta$ the location of joint is at $\left(\tau, \theta^{\prime}\right)=$ $\left(0, \delta^{\prime}\right)$. In this way the induced metric on the joint and scalar curvature tensor would be

$d \hat{s}^{2}=\tilde{L}^{2} \cot ^{2} \delta^{\prime} d \Omega_{d-2}^{2}, \quad \hat{\mathcal{R}}=(d-2)(d-3) \frac{\tan ^{2} \delta^{\prime}}{\tilde{L}^{2}}$.

Also for a joint between two null boundaries in this coordinate

$a=-\log \left|\frac{k_{1} \cdot k_{2}}{2}\right|=-\log \left(\alpha_{1} \alpha_{2} \sin ^{2} \delta^{\prime}\right)$.

By substituting the above values into the joint action (3.5), finally, we obtain

$$
\begin{aligned}
I_{\text {joint }}= & \frac{2 \tilde{L}^{d-2} \Omega_{d-2}}{\kappa^{2} \tan ^{d-2} \delta^{\prime}} \log \left(\sqrt{\alpha_{1} \alpha_{2}} \sin \delta^{\prime}\right) \\
& \times\left[\lambda_{e f f}+2 a_{3} \frac{(d-3)(d-2)}{\tilde{L}^{2}} \tan ^{2} \delta^{\prime}\right] .
\end{aligned}
$$

We can write the above expression in terms of original cutoff by using $\delta=\frac{2 \tilde{L} \sin \delta^{\prime}}{1+\cos \delta^{\prime}}$. After expansion around $\delta=0$ we find

$$
\begin{aligned}
I_{\text {joint }}= & -\frac{2 \tilde{L}^{d-2} \Omega_{d-2}}{\kappa^{2}}\left(\lambda_{e f f} N_{1}+2 a_{3} \frac{(d-3)(d-2)}{\tilde{L}^{2}} N_{2}\right) \\
& \times \log \left(\frac{\tilde{L}}{\sqrt{\alpha_{1} \alpha_{2}} \delta}\right)+\cdots, \\
N_{1}= & \sum_{n=0} \frac{\left(-\frac{1}{4}\right)^{n} \Gamma(d-1)}{\Gamma(d-1-n) \Gamma(n+1)}\left(\frac{\tilde{L}}{\delta}\right)^{d-2-2 n}, \\
N_{2}= & \sum_{n=0} \frac{\left(-\frac{1}{4}\right)^{n} \Gamma(d-3)}{\Gamma(d-3-n) \Gamma(n+1)}\left(\frac{\tilde{L}}{\delta}\right)^{d-4-2 n},
\end{aligned}
$$

where dots represent the power expansion terms. Therefore the universal term for GQC from the joint term for even $d$ dimensional space-time is given by

$$
\begin{aligned}
C_{\text {joint }}^{\text {univ }} & =(-1)^{\frac{d-2}{2}} b_{d} \log \left(\frac{\tilde{L}}{\sqrt{\alpha_{1} \alpha_{2}} \delta}\right), \\
b_{d} & =-\frac{2 \tilde{L}^{d-2} \Omega_{d-2}}{\pi \hbar \kappa^{2}} \frac{\Gamma(d-1)}{2^{d-2} \Gamma\left(\frac{d}{2}\right)^{2}}\left(\lambda_{e f f}-\frac{(d-2)^{2} a_{3}}{2 \tilde{L}^{2}}\right) .
\end{aligned}
$$

We can also find the universal terms, which obtain from the computation of the bulk action on WDW patch. In $d$ dimensional space-time these terms are computed in [47]

$C_{\text {bulk }}^{\text {univ }}= \begin{cases}(-1)^{\frac{d-3}{2}} \frac{2}{\hbar} a_{d}^{*} & \text { for odd d }, \\ (-1)^{\frac{d-4}{2}} \frac{4}{\pi \hbar} a_{d}^{*} \log \frac{\tilde{L}}{\delta} & \text { for even d, }\end{cases}$

where $a_{d}^{*}$ is the $a$-anomaly, and for GQC theory it is equal to (see [48] for more discussions)

$$
\begin{aligned}
a_{d}^{*}= & \left.\frac{\pi^{\frac{d-1}{2}} \tilde{L}^{d}}{(d-1) \Gamma\left(\frac{d-1}{2}\right)} \mathcal{L}_{\text {bulk }}\right|_{A d S} \\
= & \frac{\pi^{\frac{d-1}{2}} \tilde{L}^{d-4}}{\kappa^{2} \Gamma\left(\frac{d-1}{2}\right)} \\
& \times\left(\tilde{L}^{2}-2\left((d-1)\left(d a_{1}+a_{2}\right)+a_{3}(d-2)(d-3)\right)\right) .
\end{aligned}
$$




\section{Conclusion and discussion}

In this paper we use the CA conjecture (1.2) for studying the holographic complexity. In the bulk space-time, we assume a general quadratic action that includes both Riemann and Ricci curvature tensors up to the quadratic terms (2.1). This form of action allows us to generalize the ideas around the holographic complexity when one takes into account the higher curvature theories of gravity in $d$ dimensional spacetime.

In Sect. 2 we examine the proposal of growth bound on complexity for two types of charged and neutral black holes of GQC that are asymptotically AdS space-time. In Sect. 2.2 we first find a $U(1)$ charged black hole solution and then in Sect. 2.3 we obtain the action growth rate $(2.15)$ by computing the bulk and boundary actions on WDW patch at late-time approximation. Our final result (2.16) confirms the proposal in [17], i.e. we can write the total action growth as a difference between the value of $M-\mu Q$ on the outer and inner horizons of the black hole. This result has been already reported for various theories of gravity [26], and here we observe that this bound is preserved for general quadratic curvature theory of gravity.

Despite this, the case of the neutral black hole is more challenging. The reason is the existence of the singularity as one of the surface boundaries of the WDW patch. In Sect. 2.4 we learn how we can guess and compute the singularity structure of geometry in GQC theory from a particular class of solutions. Using this, in Sect. 2.5 we compute the action growth rate. We show that for special cases such as $a_{3}=0$ or $d=4$ where the singularity is located at $r_{s}=0$, the singularity does not produce any divergences and the Lloyd's bound saturates at $2 M$.

The above results hold when the singularity point is located behind the horizon(s). A numerical survey shows that, for certain regions of the parameters of the theory a second singularity appears at $r_{s} \neq 0$. For neutral black holes this changes the expected proposal of [17]. Moreover for charged black holes when this singularity is behind the inner horizon, the action growth rate saturates the Lloyd's bound. But when it is located between the two horizons then it leads to a result other than the expected Lloyd's bound.

It is worth reminding that, even though higher order curvature theories of gravity may contain the ghost modes, as indicated in [30] it can be shown that the strong energy condition is a sufficient condition to ensure the bound inequality, therefore in this paper, we suppose this energy condition everywhere, (for more details on energy conditions of the GQC see [48]).

In Sect. 3 we look into another interesting subject in the context of holographic complexity and compute the universal terms that appear in the divergent part of $C_{A}$ for the GQC theory of gravity. Usually, there are two types of these uni- versal terms, one from the joint terms of regularized WDW patch and one from the bulk action. Using a simple trick, by introducing an effective GH term (3.4), we find the joint terms from the techniques in paper [47]. In that paper, the joint terms were calculated for Lovelock theory. Although the GQC cannot be written in terms of the Lovelock theory, its GH terms as we mentioned, are compatible with those in [47] technique. In this way, we find the universal terms (3.14b) and (3.16).

Acknowledgements We would like to thank M. Siahvoshan for useful discussion. This work is supported by Ferdowsi University of Mashhad under the grant $2 / 52193$.

Data Availability Statement This manuscript has no associated data or the data will not be deposited. [Authors' comment: All data is produced by Mathematica software.]

Open Access This article is licensed under a Creative Commons Attribution 4.0 International License, which permits use, sharing, adaptation, distribution and reproduction in any medium or format, as long as you give appropriate credit to the original author(s) and the source, provide a link to the Creative Commons licence, and indicate if changes were made. The images or other third party material in this article are included in the article's Creative Commons licence, unless indicated otherwise in a credit line to the material. If material is not included in the article's Creative Commons licence and your intended use is not permitted by statutory regulation or exceeds the permitted use, you will need to obtain permission directly from the copyright holder. To view a copy of this licence, visit http://creativecomm ons.org/licenses/by/4.0/.

Funded by $\mathrm{SCOAP}^{3}$.

\section{A Useful relations}

Using the anzats in (2.7) we find the following useful expressions for computing the bulk Lagrangian (2.1)

$$
\begin{aligned}
R= & -\frac{r^{-2}}{2 f_{1}^{2}}\left(2(d-2) f_{1}^{2}\left((d-3)\left(f_{2}-1\right)+r f_{2}^{\prime}\right)\right. \\
& -r^{2} f_{2} f_{1}^{\prime 2}+r f_{1} \\
& \left.\times\left(r f_{1}^{\prime} f_{2}^{\prime}+2\left((d-2) f_{1}^{\prime}+r f_{1}^{\prime \prime}\right) f_{2}\right)\right)
\end{aligned}
$$

$$
\begin{aligned}
R_{\mu \nu} R^{\mu \nu}= & \frac{r^{-4}}{8 f_{1}^{4}}\left(2 ( d - 2 ) f _ { 1 } ^ { 4 } \left(4(d-3)^{2}\left(f_{2}-1\right)^{2}\right.\right. \\
& \left.+4(d-3)\left(f_{2}-1\right) r f_{2}^{\prime}+(d-1) r^{2}{f_{2}^{\prime}}^{2}\right) \\
& +r^{4} f_{2}^{2} f_{1}^{\prime 4}-2 r^{3} \\
& \times\left(f_{1}^{\prime}\left((d-2) f_{2}+r f_{2}^{\prime}\right)+2 r f_{2} f_{1}^{\prime \prime}\right) \\
& \times f_{1} f_{2} f_{1}^{\prime 2}+2(d-2) r f_{1}^{3} \\
& \times\left(2 r^{2} f_{2} f_{2}^{\prime} f_{1}^{\prime \prime}+f_{1}^{\prime}\right. \\
& \left.\times\left(4(d-3)\left(f_{2}-1\right) f_{2}+2 r f_{2} f_{2}^{\prime}+r^{2} f_{2}^{\prime 2}\right)\right) \\
& +f_{1}^{2} r^{2}\left(f_{1}^{\prime 2}\left(2(d-2)(d-1) f_{2}^{2}+r^{2}{f_{2}^{\prime}}^{2}\right)\right.
\end{aligned}
$$




$$
\begin{aligned}
& +4 r f_{2} f_{1}^{\prime}\left((d-2) f_{2}+r f_{2}^{\prime}\right) f_{1}^{\prime \prime} \\
& \left.\left.+4 r^{2} f_{2}^{2} f_{1}^{\prime \prime 2}\right)\right), \\
R_{\mu \nu \alpha \beta} R^{\mu \nu \alpha \beta}= & \frac{1}{4 r^{4} f_{1}^{4}}\left(r^{4} f_{1}^{\prime 4} f_{2}^{2}+4(d-2) f_{1}^{4}\right. \\
& \times\left(2(d-3)\left(f_{2}-1\right)^{2}+r^{2} f_{2}^{\prime 2}\right) \\
& -2 r^{4} f_{1} f_{1}^{\prime 2} f_{2}\left(f_{1}^{\prime} f_{2}^{\prime}+2 f_{1}^{\prime \prime} f_{2}\right) \\
& +f_{1}^{2} r^{2}\left(f_{1}^{\prime 2}\left(4(d-2) f_{2}^{2}+r^{2} f_{2}^{\prime 2}\right)\right. \\
& \left.\left.\times+4 r^{2} f_{1}^{\prime} f_{1}^{\prime \prime} f_{2} f_{2}^{\prime}+4 r^{2} f_{1}^{\prime \prime 2} f_{2}^{2}\right)\right) .
\end{aligned}
$$

$$
\begin{aligned}
\alpha_{4}= & 2 \Lambda_{0}\left((d-2)^{2}(d-1)+2 d \Lambda_{0}\right. \\
& \left.\times\left(a_{3}(d-3)(d-2)+a_{2}(d-1)+a_{1}(d-1) d\right)\right), \\
\alpha_{5}= & (d-3)(d-2)^{3}(d-1) q^{4} \\
& \times\left(a_{3}\left(9 d^{2}-45 d+56\right)-a_{1}(d-4)\left(12 d^{2}-45 d+43\right)\right. \\
& \left.-a_{2}\left(-39+53 d-23 d^{2}+3 d^{3}\right)\right) .
\end{aligned}
$$

The coefficients of boundary action growth in Eq. (2.14) are

The sum of GH terms (2.2) and (2.5) are given by

$$
\begin{aligned}
\left(g^{\alpha \beta}\right. & \left.+\frac{\partial L^{R i c c i}}{\partial R_{\alpha \mu \beta \nu}} n_{\mu} n_{\nu}\right) K_{\alpha \beta} \\
= & \frac{f_{2}^{\frac{1}{2}}}{2 r f_{1}}\left(2(d-2) f_{1}+r f_{1}^{\prime}\right)+\frac{f_{2}^{\frac{1}{2}}}{4 f_{1}^{3} r^{3}} \\
\quad & \times\left(\left(2 a_{1}+a_{2}\right) r^{3} f_{2} f_{1}^{\prime 3}-2(d-2) f_{1}^{3}\right. \\
& \times\left(2(d-3)\left(a_{2}+2 a_{1}(d-2)\right)\left(f_{2}-1\right)\right. \\
& \left.+\left(4 a_{1}(d-2)+a_{2}(d-1)\right) r f_{2}^{\prime}\right) \\
& -\left(2 a_{1}+a_{2}\right) r^{3} f_{1} f_{1}^{\prime}\left(f_{1}^{\prime} f_{2}^{\prime}+2 f_{2} f_{1}^{\prime \prime}\right)-2(d-2) f_{1}^{2} r \\
& \times\left(f _ { 1 } ^ { \prime } \left(\left(4 a_{1}+a_{2}\right) r f_{2}^{\prime}-2 a_{1}(d-3)\right.\right. \\
& \left.\left.\left.+\left(a_{2}+(6 d-14) a_{1}\right) f_{2}\right)+\left(4 a_{1}+a_{2}\right) r f_{2} f_{1}^{\prime \prime}\right)\right) .
\end{aligned}
$$

The GH term (2.4) has the following contribution

$$
\begin{aligned}
& 2 \mathcal{G}_{a b} K^{a b}+\frac{1}{3}\left(K^{3}-3 K K_{a b} K^{a b}+2 K_{a b} K^{b c} K_{c}{ }^{a}\right) \\
& =\frac{(d-3)(d-2) f_{2}^{\frac{1}{2}}}{6 f_{1} r^{3}} \\
& \quad \times\left(2(d-4) f_{1}\left(f_{2}-3\right)+3 r\left(f_{2}-1\right) f_{1}^{\prime}\right) .
\end{aligned}
$$

\section{B Action growth coefficients}

The coefficients of bulk action growth in Eq. (2.13) are

$$
\begin{aligned}
\alpha_{1}= & (d-3)(d-2) q^{2} \\
& \times\left((d-2)^{2}(d-1)+2(d-3) \Lambda_{0}\left(-2 a_{3}(d-4)(d-2)\right.\right. \\
& \left.\left.+2 a_{1}(d-4)(d-1)(2 d-3)+a_{2}\left(6+(d-4) d^{2}\right)\right)\right), \\
\alpha_{2}= & -(d-3)(d-2)^{3}(d-1)^{2} \\
& \times\left((d-4)\left(4 a_{1}(d-1)+a_{2} d\right) q^{2}-a_{3}(d-2) m^{2}\right), \\
\alpha_{3}= & 2(d-3)(d-2)^{4}(d-1) q^{2} m \\
& \times\left(a_{3}(10-4 d)+4 a_{1}(d-4)(d-1)+a_{2}(d-4) d\right),
\end{aligned}
$$

$$
\begin{aligned}
\beta_{1}= & 2(d-3)(d-2)^{3}(d-1) \\
& \times\left(3 a_{1}(d-4)\left(31-38 d+11 d^{2}\right)\right. \\
& +3 a_{2}\left(9+4 d-6 d^{2}+d^{3}\right) \\
& \left.-a_{3}(3 d-7)\left(d^{2}+3 d-16\right)\right) q^{4}, \\
\beta_{2}= & 4(3 d-7) \Lambda_{0} \\
& \times\left(3(d-2)^{2}(d-1)-2 a_{3}(d-8)(d-3)(d-2) \Lambda_{0}\right. \\
& \left.+6(d-1) d\left(a_{2}+a_{1} d\right) \Lambda_{0}\right), \\
\beta_{3}= & 2(d-2)^{3}(3 d-7) \\
& \times(-3(d-2)(d-1) \\
& \left.+4\left(a_{3}(d-4)(d-3)-3(d-1)\left(a_{2}+d a_{1}\right)\right) \Lambda_{0}\right), \\
\beta_{4}= & -8 a_{3}(d-4)(d-3)(d-2)^{4}(d-1)(3 d-7), \\
\beta_{5}= & (d-2)^{2}(d-1)(3 d-7) m \\
& \times(3(d-2)(d-1) \\
& \left.+4\left(a_{3}(d-3)(d-2)+3(d-1)\left(a_{2}+d a_{1}\right)\right) \Lambda_{0}\right), \\
\beta_{6}= & 4 a_{3}(d-4)(d-3)(d-2)^{4}(d-1)(3 d-7) m, \\
\beta_{7}= & -2(d-2)(3 d-7) q^{2} \\
& \times\left(3(d-2)^{2}(d-1)+2(d-3)\right. \\
\beta_{9}= & \times\left(-2 a_{3}(d-4)(d-2)(d+1)\right. \\
& \times\left(12 a_{1}(d-4)(d-1)+3 a_{2}(d-4) d-2 a_{3}\left(d^{2}-7\right)\right) m q^{2} . \\
& +6 a_{1}(d-4)(d-1)(3 d-5) \\
& \left.\left.+3 a_{2}\left(-6+22 d-16 d^{2}+3 d^{3}\right)\right) \Lambda_{0}\right), \\
& -2(d-3)(d-2)^{3}(d-1)(3 d-7) \\
& \left(-3\left(2 a_{1}(d-4)(3 d-5)+a_{2}\left(18-16 d+3 d^{2}\right)\right) q^{2}\right. \\
& \left.(d-2)\left((d-1) m^{2}+2(d-4) q^{2}\right)\right), \\
& (d-2)^{3}(d-1)(3 d-7) \\
& (d-3)
\end{aligned}
$$

\section{References}

1. S. Ryu, T. Takayanagi, Holographic derivation of entanglement entropy from AdS/CFT. Phys. Rev. Lett. 96, 181602 (2006). arXiv:hep-th/0603001 
2. S. Ryu, T. Takayanagi, Aspects of holographic entanglement entropy. JHEP 08, 045 (2006). arXiv:hep-th/0605073

3. L. Susskind, Entanglement is not enough. Fortsch. Phys. 64, 49 (2016). arXiv:1411.0690 [hep-th]

4. J. Watrous, Quantum computational complexity, encyclopedia of complexity and systems science, ed. by R.A. Meyers (2009), pp. 7174-7201. arXiv:0804.3401 [quant-ph]

5. S. Gharibian, Y. Huang, Z. Landau, S.W. Shin, Quantum hamiltonian complexity. Found. Trends Theor. Comput. Sci. 10, 159-282 (2015). arXiv:1401.3916 [quant-ph]

6. T.J. Osborne, Hamiltonian complexity. Rep. Prog. Phys. 75, 022001 (2012). arXiv:1106.5875 [quant-ph]

7. L. Susskind, Computational complexity and black hole horizons. Fortsch. Phys. 64, 24-43 (2016). arXiv:1402.5674 [hep-th]

8. L. Susskind, Addendum to: Computational complexity and black hole horizons. Fortsch. Phys. 64, 44 (2016). arXiv:1403.5695 [hepth]

9. D. Stanford, L. Susskind, Complexity and shock wave geometries. Phys. Rev. D 90, 126007 (2014). arXiv:1406.2678 [hep-th]

10. A.R. Brown, D.A. Roberts, L. Susskind, B. Swingle, Y. Zhao, Holographic complexity equals bulk action? Phys. Rev. Lett. 116(19), 191301 (2016). arXiv:1509.07876 [hep-th]

11. A.R. Brown, D.A. Roberts, L. Susskind, B. Swingle, Y.Zhao, Complexity, action, and black holes. Phys. Rev. D 93(8), 086006 (2016). arXiv:1512.04993 [hep-th]

12. L. Lehner, R.C. Myers, E. Poisson, R.D. Sorkin, Gravitational action with null boundaries. Phys. Rev. D 94(8), 084046 (2016). arXiv:1609.00207 [hep-th]

13. J.M. Maldacena, Eternal black holes in anti-de Sitter. JHEP 0304, 021 (2003). arXiv:hep-th/0106112

14. J. Maldacena, L. Susskind, Cool horizons for entangled black holes. Fortsch. Phys. 61, 781 (2013). arXiv:1306.0533 [hep-th]

15. S. Chapman, H. Marrochio, R.C. Myers, Complexity of Formation in Holography. JHEP 01, 062 (2017). arXiv:1610.08063 [hep-th]

16. S. Lloyd, Ultimate physical limits to computation. Nature (London) 406, 1047 (2000)

17. R.G. Cai, S.M. Ruan, S.J. Wang, R.Q. Yang, R.H. Peng, Action growth for AdS black holes. JHEP 1609, 161 (2016). arXiv: 1606.08307 [gr-qc]

18. H. Huang, X.H. Feng, H. Lu, Holographic complexity and two identities of action growth. Phys. Lett. B 769, 357 (2017). arXiv:1611.02321 [hep-th]

19. R. Cai, M. Sasaki, S. Wang, Action growth of charged black holes with a single horizon. Phys. Rev. D 95(12), 124002 (2017). arXiv: 1702.06766 [gr-qc]

20. D. Carmi, S. Chapman, H. Marrochio, R.C. Myers, S. Sugishita, On the time dependence of holographic complexity. JHEP 11, 188 (2017). arXiv:1709.10184 [hep-th]

21. K. Goto, H. Marrochio, R.C. Myers, L. Queimada, B. Yoshida, Holographic complexity equals which action? JHEP 02, 160 (2019). arXiv:1901.00014 [hep-th]

22. Z. Fan, M. Guo, Holographic complexity and thermodynamics of AdS black holes. Phys. Rev. D 100, 026016 (2019). arXiv:1903.04127 [hep-th]

23. J. Jiang, X. Li, Adjusted complexity equals action conjecture. Phys. Rev. D 100(6), 066026 (2019). arXiv:1903.05476 [hep-th]

24. Z. Fan, H. Liang, Time dependence of complexity for Lovelock black holes. Phys. Rev. D 100(8), 086016 (2019). arXiv: 1908.09310 [hep-th]

25. J. Jiang, M. Zhang, Holographic complexity of the electromagnetic black hole. Eur. Phys. J. C 80(2), 85 (2020). arXiv:1905.07576 [hep-th]

26. J. Jiang, Action growth rate for a higher curvature gravitational theory. Phys. Rev. D 98(8), 086018 (2018). arXiv: 1810.00758 [hepth]
27. W. Sun, X.H. Ge, Complexity growth rate, grand potential and partition function. arXiv:1912.00153 [hep-th]

28. J. Couch, W. Fischler, P.H. Nguyen, Noether charge, black hole volume, and complexity. JHEP 03, 119 (2017). arXiv:1610.02038 [hep-th]

29. Z. Fan, M. Guo, On the Noether charge and the gravity duals of quantum complexity. JHEP 08, 031 (2018). arXiv:1805.03796 [hep-th]

30. R.Q. Yang, Strong energy condition and complexity growth bound in holography. Phys. Rev. D 95(8), 086017 (2017). arXiv: 1610.05090 [gr-qc]

31. J. Jiang, B.X. Ge, Investigating two counting methods of the holographic complexity. Phys. Rev. D 99(12), 126006 (2019). arXiv:1905.08447 [hep-th]

32. E. Dyer, K. Hinterbichler, Boundary terms, variational principles and higher derivative modified gravity. Phys. Rev. D 79, 024028 (2009). arXiv:0809.4033 [gr-qc]

33. A. de la Cruz-Dombriz, A. Dobado, A.L. Maroto, Black holes in f(R) theories. Phys. Rev. D 80, 124011 (2009). arXiv:0907.3872 [gr-qc] [Erratum: Phys. Rev. D 83, 029903 (2011)]

34. A. Guarnizo, L. Castaneda, J.M. Tejeiro, Boundary term in metric $\mathrm{f}(\mathrm{R})$ gravity: field equations in the metric formalism. Gen. Relativ. Gravity 42, 2713 (2010). arXiv:1002.0617 [gr-qc]

35. T.S. Bunch, Surface terms in higher derivative gravity. J. Phys. A 14, L139 (1981)

36. R.C. Myers, Higher-derivative gravity, surface terms, and string theory. Phys. Rev. D 36, 392 (1987)

37. T. Padmanabhan, D. Kothawala, Lanczos-Lovelock models of gravity. Phys. Rept. 531, 115 (2013). arXiv:1302.2151 [gr-qc]

38. P. Bueno, P.A. Cano, A.O. Lasso, P.F. Ramirez, f(Lovelock) theories of gravity. JHEP 1604, 028 (2016). arXiv:1602.07310 [hep-th]

39. A. Teimouri, S. Talaganis, J. Edholm, A. Mazumdar, Generalised boundary terms for higher derivative theories of gravity. JHEP 1608, 144 (2016). arXiv:1606.01911 [gr-qc]

40. N. Deruelle, M. Sasaki, Y. Sendouda, D. Yamauchi, Hamiltonian formulation of $\mathrm{f}$ (Riemann) theories of gravity. Prog. Theor. Phys. 123, 169-185 (2010). arXiv:0908.0679 [hep-th]

41. M. Alishahiha, A. Faraji Astaneh, A. Naseh, M.H. Vahidinia, On complexity for $F(R)$ and critical gravity. JHEP 05, 009 (2017). arXiv:1702.06796 [hep-th]

42. Y. Ding, T. Wang, Action growth rates of black holes in the ChernSimons modified gravity. arXiv:1811.08113 [hep-th]

43. J. Jiang, H. Zhang, Surface term, corner term, and action growth in $F\left(R_{a b c d}\right)$ gravity theory. Phys. Rev. D 99(8), 086005 (2019). arXiv:1806.10312 [hep-th]

44. P.A. Cano, R.A. Hennigar, H. Marrochio, Complexity growth rate in lovelock gravity. Phys. Rev. Lett. 121(12), 121602 (2018). arXiv:1803.02795 [hep-th]

45. Y. An, R. Cai, Y. Peng, Time dependence of holographic complexity in Gauss-Bonnet gravity. Phys. Rev. D 98(10), 106013 (2018). arXiv: 1805.07775 [hep-th]

46. D. Carmi, R.C. Myers, P. Rath, Comments on holographic complexity. JHEP 03, 118 (2017). arXiv:1612.00433 [hep-th]

47. P.A. Cano, Lovelock action with nonsmooth boundaries. Phys. Rev. D 97(10), 104048 (2018). arXiv:1803.00172 [gr-qc]

48. A. Ghodsi, M. Siahvoshan, A holographic study of the $a$-theorem and RG Flow in general quadratic curvature gravity. Eur. Phys. J. C 79(10), 820 (2019). arXiv:1907.03497 [hep-th]

49. A. Ghodsi, F. Najafi, Ricci cubic gravity in d dimensions, gravitons and SAdS/Lifshitz black holes. Eur. Phys. J. C 77(8), 559 (2017). arXiv:1702.06798 [hep-th] 